\title{
Analysis of high-intensity physical activity biological feasibility within the framework of children health training
}

\author{
Artem Moment", Viktor Nemerovsky, Elena Karpenko, Lena Rubenkova, and Aleksey Nikitin \\ Pskov State University, 180007, Pskov, Russia
}

\begin{abstract}
Epidemiological surveys reflect a negative trend in functional indicators for children's physical health. In recent surveys it is experimentally proved that unlike other methods of training, high-intensity interval training (HIIT) produces prevailing positive effect onto the indicators of physical and functional condition of children with poor health. However, the review of studies suggests that the stated idea turns to be controversial. There is the evidence of possible negative effects of intense physical activity due to the low level biological reliability of adaptation mechanisms and other anatomical and physiological characteristics of children. Therefore, it is necessary to provide a scientifically based conceptual model of timeline for children's health training in which the central health impetus will be HIIT.
\end{abstract}

\section{Introduction}

In the sphere of physical education and healthcare specialists pay close attention to the issue of maintenance and improvement of health of the younger generation and this question has not lost its relevance for several decades yet. Many epidemiological studies report a current negative tendency in functional indicators of children's physical health $[1,2,3]$.

Among all functional conditions, which are the indicators of weakened health, a special place is occupied by the disorders in musculoskeletal system [4]. Low indicators of strength abilities of the muscle groups, which are involved in the maintenance of orthograde body posture and performing basic locomotion result in various combinations of postural disorders in saggital and frontal plane $[5,6,7]$. The main factor adversely affecting the strength abilities of children is the lack of rationally organized motor activity $[6,8,9]$. A low level of motor activity along with excessive highcarbohydrate meals have resulted in the global problem of obesity for both children and adults $[3,8]$.

Currently, researchers have proved the effectiveness of high-intensity physical activity for child's organism within the framework of health training sessions. A direct dependence has been established between the intensity and the volume of training load on power outcome performnce and its contribution to improving the functional condition and motor fitness of children [10]. However, the scientific literature has accumulated a lot of data on the ambiguity of such a strategy. In the long run, continuous high-intensity loads on the child's body often lead to violations in adaptation mechanisms [11, 12], the appearance of muscle imbalance and disorders in motor automatisms [2, 5], and a high risk of injuries $[13,14]$.

The aim of the study is to analyze the biological feasibility of high intensity physical activity in the framework of children health training.

\section{Materials and methods}

Survey and analysis of the scientific literature on the adaptation of the child's body to the systematic effects of physical activity of various orientations and intensities.

\section{Results and discussion}

Many scientific studies are devoted to the methodology of children physical training. Traditionally, in the Russian scientific literature on sports physiology and the theory and methods of physical education, scientifically substantiated proofs of the large volume and intensity physical activity negative influence on the rate of the second childhood period general development of children were proposed. At the moment, many modern Russian and foreign studies challenge long-established canons $[10,15,16]$. For instance, it was reported that under the influence of training on power output performance, the concentration of insulin-like growth factor-1 in the blood serum increases significantly, stimulating

\footnotetext{
${ }^{*}$ Corresponding author: ar.moment@yandex.ru
} 
growth and increase in bone density [17]. Children and adolescents possess great potential to improve physical and functional abilities. However, children have a low biological reliability of adaptive mechanisms. One of the main systems, which acts as a limiting factor in the development of adaptive mechanisms, is the cardiovascular system [12].

Foreign studies consider a strong health-improving effect after high-intensity interval training (HIIT) compared with continuous training of medium intensity (MICT - moderate-intensity continuous training), resistance training (RT), and high-volume hypertrophic training (HVT - high volume training).

In the load on power output performance phase, when employing the HIIT protocol, the load is $80-100 \%$ of the maximum heart rate (HR max) and maximum oxygen consumption (VO2max); when implementing the MICT protocol, the load during training session is $55-70 \%$ of HR max, or about $40-60 \%$ from VO2max. RT parameters to a lesser extent impose requirements on the cardio-respiratory system. The intensity of the target physical activity is calculated as a percentage of the repeated maximum in this movement and ranges from 50-60\% to $95-100 \%$. It should be noted that for children of primary school age it is not recommended to employ loads on power output performance of maximum and submaximum intensity $[13,18]$. It is known that the increase in indicators among children of prepubertal age occurs mainly due to the initial improvement in intermuscular coordination (with moderate intensity of power load relative to a subsequent increase in muscle activation (at high intensity of the power load relative to 1PM), thereby increasing the indicators of power endurance and relative strength. Hypertrophic strength training intervention, involving the implementation of strength exercises in the anaerobic-lactate mode, has little effect on the growth of muscle mass in children [13]. However, the optimal volume and duration of the of anaerobic glycolysis metabolites effect contributes to increased activity of metabolic processes in muscle cells, in particular by improving the throughput of cell membranes. Thus, a strong metabolic effect is produced without significant stress on the cardiovascular system.

The duration of a HIIT training session is from 4 to 20-30 minutes, MICT lasts 20-60 minutes, RT is on average 4060 minutes, HVT is not less than 40 minutes.

The HIIT composition involves the alternation of several bursts of high-intensity exercises with exercises of low or moderate intensity in a strictly defined time frame (for example: 30 seconds of high-intensity workout is followed by 30 seconds of relatively low-intensity exercises. It promotes active recovery).

A meta-analysis suggests that HIIT is superior to MICT on the change in VO2max in children and adolescents. The presumptive physiological mechanism of such adaptation is described in the trial. There the subjects for two weeks at six training sessions performed the same exercises for the leg muscles. For one leg HIIT protocol was employed, and for another - MICT protocol. The analysis showed a more significant increase in the number of mitochondria, an increase in the maximum activity of the Krebs oxidative enzyme cycle (citrate synthase), and an improvement in the activation of type II muscle fibers during the exercises employing the HIIT protocol [15].

Another research compared the effects of implementation of HIIT and MICT protocols for adolescent cardiac activity. During a five-week experiment, which was based on an analysis of heart rate variability, the prevailing advantageous effect of the implementation of HIIT protocol on adolescent cardiac activity by means of increased vagal activity was proved [19].

Another experiment examined the effects of HIIT protocols and high volume hypertrophic strength training (HVT high volume training) on biological stress markers in the saliva of teenagers aged $14 \pm 1$ years playing sports. The levels of cortisol, testosterone, alpha-amylase were measured; markers of metabolic and cardiorespiratory stress: blood lactate, blood $\mathrm{pH}$, heart rate, oxygen consumption (VO2), etc. Levels of cortisol, testosterone, and alpha-amylase were determined before the exercises, immediately after, and then at 30, 60, 180 minutes after the performance of the designed protocols. Markers of metabolic and cardiorespiratory stress were recorded during the exercise. The researchers noted a significant increase in cortisol levels at 30 and 60 minutes after the implementation of HIIT protocol compared to the HVT protocol. However, at 180 minutes after the implementation of HIIT and HVT protocol, cortisol levels decreased below baseline. Testosterone levels increased immediately after the implementation of HVT protocol, whereas after the HIIT protocol, testosterone began to increase actively only after 30 minutes. Apparently, this is due to the high concentration of cortisol immediately after the implementation of HIIT protocol. Metabolic and cardiorespiratory stress was higher in HIIT session. Based on the data, the researchers conclude that there are no serious acute catabolic effects of implementation of HIIT and HVT, and, accordingly, justify their safety for human health [20].

A meta-analysis suggests the positive effects of implementation of HIIT protocols on glucose and lipid metabolism, body composition and blood pressure in obese children. A decrease in insulin resistance in this group of children was detected. However, the researchers consider the effectiveness of the implementation of HIIT protocol in relation to MICT to be ambiguous [16]. Another group of researchers found a significant decrease in waist circumference and heart rate at rest in obese children using the combination of HIIT + MICT protocols. However, implementation of HIIT protocols has a greater effect on lowering fasting glucose, c-reactive protein and increasing VO2max relative to MICT and HIIT + MICT [21]. Younger obese pupils aged 7-9 years participated in a research of the effectiveness of the implementation of HIIT protocols on weight loss. It also showed a significant decrease in BMI in experimental group of children [22].

Scientists from Japan evaluated the impact of the four-week HIIT program with a frequency of training sessions 3 times a week on the motor and cognitive abilities of children aged 8-12 years old. The duration of the HIIT protocol varied linearly from 8 minutes in the first week of training to 10 minutes in the last week. Peak load was $\geq 85 \%$ of HRmax. The load ratio of high and low intensity was 30/30 seconds. Exercise complexes included: running 13 and 26 meters, various variants of vertical jumps, exercises for the muscles of the body. Before the target load, a warm-up was 
performed for 10 minutes, and after a cool-down lasting 5 minutes. A significant increase in cognitive abilities and indicators of speed and power endurance is shown. Researchers note the prospect of introducing HIIT protocols into the school curriculum for physical education due to its effectiveness and short duration of classes in relation to moderateintensity training [23].

There are many ways to vary and modify implementation of HIIT. So during a twelve-week experiment, the effectiveness of the implementation of HIIT and HIIT + P protocols (with the additional inclusion of plyometric exercises) was compared to reduce BMI, glucose and leptin levels in blood plasma, and the height of the squat jump. The analysis of the experimental results showed a more significant decrease in BMI, plasma glucose and the height of the squat jump in the group of adolescents who employed HIIT + P protocol [24]. In spite of the shown advantageous positive healing effect of the HIIT + P protocol compared to HIIT, there are still doubts about the safety of using plyometric exercises for children and adolescents who are overweight. Jump-off and landing can cause displacement of the pelvic bones and improper fusion, especially if the load is asymmetric. Recent studies have shown that younger pupils often have muscle imbalances that negatively affect the stability of the spine and pelvic bones [25]. Additional jumping stress, in this case, significantly increases the risk of injury and contributes to the formation of abnormalities of gait and posture.

A retrospective analysis showed a direct correlation between the introduction of HIIT protocols in modern fitness and the increase in injuries, especially sprains and deformations of the knee and ankle joints. Researchers make a logical conclusion that before implementing the HIIT protocols in the training process, it is necessary to first strengthen the muscles, tendons and ligaments, and also pay special attention to neuromuscular training [14]. Preliminary strength training within the framework of the RT protocol allows to create adequate prerequisites for strengthening muscles and ligaments, increase bone density and help improve neuromuscular connections $[18,26]$. It in combination with cardio loads of low and moderate intensity, will contribute to the smooth adaptation of the cardiovascular system to high intensity loads within the framework of the HIIT protocol.

In all the experiments analyzed, a HIIT block was employed for 4-12 weeks. The dynamics of physical activity throughout the block was linear in nature. It, taking into account the ultimate or near-ultimate loads in the framework of each training session, is not the preferred model for the periodization of the load, especially for health training.

\section{Conclusions}

Despite the advantages of the HIIT protocols implementation which were reported in sports and health training with children. It should be kept in mind that rapid anthropometric and functional changes (caused by intense load on power outcome performance) occur in the organism of a child against the background of increased energy demand for the implementation of plastic processes. The low biological reliability of the adaptation mechanisms in combination with the anatomical and physiological characteristics of children does not allow to fully reveal the high level of fitness potential of HIIT implementation, as an independent and self-sufficient training unit. So, for example, in the framework of sports training, there is timing of the training process. There high intensity interval loads can be an adequate component of the young athletes training session accompanied by the preparatory period. In it the technical, strength and functional base is laid, and the subsequent transitional (recovery) period contributing to the replenishment adaptive reserves of the body.

HIIT definitely triggers a strong central-nervous and metabolic response in children. It in turn creates a comparable adaptive stimulus that improves cardio-respiratory and strength endurance, increases cognitive abilities and decreases insulin resistance. However, in recent studies we haven't detected conceptual models of periodization for fitness training session, employing this technology.

As a result of the conducted analysis, a contradiction was found between the desire to get a quick fitness effect by intensifying physical activity as part of fitness training with children and the lack of designed models for timing the fitness process employing HIIT protocols.

Thus, there is a need to design a scientifically based conceptual model of the health training periodization with children. In it HIIT will be the central health stimulus.

\section{References}

1. V.N. Kochomanova, Problems and prospects of the formation of a healthy lifestyle in the information society: proceedings of the international scientific and practical conference, 100-103 (Irkutsk, 2016) (In Russ.)

2. M.O. Kuzmina, Healthcare, education, and safety, 3 (11), 68-75 (2017) (In Russ.)

3. J.A. Batch, L.A.. Baur, Med J Aust., 182(3), 130-135 (2005)

4. A.G. Sukharev, Public Health and Habitat, 2, 4-10 (2002) (In Russ.)

5. A.V. Moment, Scientific notes of the P.F. Lesgaft University: scientific and theoretical journal, 3 (157), 225-229 (SPb., P.F. Lesgaft SSU, 2018) (In Russ.)

6. V.K. Balsevich, Essays on human age kinesiology. Soviet Sport (2009) (In Russ.) 
7. I.V. Ryabova, Scientific notes of the P.F. Lesgaft University: scientific and theoretical journal, 2 (168), 300-306 (SPb., P.F. Lesgaft SSU, 2019) (In Russ.)

8. I.A. Krivolapchuk, Siberian pedagogical journal, 5, 12-25 (2016) (In Russ.)

9. E.I. Aukhadeyev, Physical education in prevention, treatment, rehabilitation: a scientific and practical journal, 34(34-35), 92-95 (2010) (In Russ.)

10. M.B. Chernova, Science without borders, 7 (12), 46-50 (2017) (In Russ.)

11. E.Kh. Sapakhova, Studies of young scientists - contribution into innovation development of Russia, 77-79 (Astrakhan, 2015) (In Russ.)

12. A.S. Kuznetsov, Z.M. Kuznetsova, Russian Journal of Physical Education and Sport, 14(4), 5-7 (2019)

13. J.M. Jegger, K. Krugger, Muscles in sport. Anatomy. Physiology. Training. Rehabilitation (Moscow, Practical Medicine, 2016) (In Russ.)

14. N.D. Rynecki, B.L. Siracuse, J.A. Ippolito, K.S. Beebe, J Sports Med Phys Fitness, 59(7), 1206-1212 (2019)

15. M. Cao, M. Quan, J. Zhuang, Int. J. Environ. Res. Public Health, 16, 1533 (2019)

16. J. Liu, L. Zhu, Y. Su, Front Physiol., 11, 214 (2020)

17. G.A. Mirzayev, Modern healthcare technologies, 3, 61-78 (2017) (In Russ.)

18. A.M. Myers, N.W. Beam, J.D. Fakhoury, Transl.Pediatr. , 6(3), 137-143 (2017)

19. A. van Biljon, A.J. McKune, K.D. DuBose, U. Kolanisi, S.J. Semple. Cardiology, 141(1), 1-8 (2018)

20. Y. Kilian, F. Engel, P. Wahl, et al. Eur J ApplPhysiol ,116, 2177-2186 (2016)

21. A. van Biljon, A.J. McKune, K.D. DuBose, U. Kolanisi, S.J. Semple, J Pediatr., 203, 325-329(2018)

22. M. Espinoza-Silva, P.Á. Latorre-Román, J. Párraga-Montilla, F. Caamaño-Navarrete, D. Jerez-Mayorga, P. Delgado-Floody, Endocrinol Diabetes Nutr., 66(10), 611-619 (2019)

23. N. Tottori, N. Morita, K. Ueta, S. Fujita, Int J Environ Res Public Health, 16(21), 4127 (2019)

24. G. Racil, H. Zouhal, W. Elmontassar, et al. ApplPhysiolNutrMetab. 41(1), 103-109 (2016)

25. A.V. Moment, Scientific notes of the P.F. Lesgaft University: scientific and theoretical journal, 3 (157), 229-232 (SPb., P.F. Lesgaft SSU, 2018) (In Russ.)

26. B. Falk, Dotan R. Harefuah, 158(8), 515-519 (2019) 